\title{
The Redshift-Dependence of Radial Acceleration: Modified Gravity versus Particle Dark Matter
}

\author{
Sabine Hossenfelder, Tobias Mistele \\ Frankfurt Institute for Advanced Studies \\ Ruth-Moufang-Str. 1, D-60438 Frankfurt am Main, Germany
}

\begin{abstract}
Modified Newtonian Dynamics has one free parameter and requires an interpolation function to recover the normal Newtonian limit. We here show that this interpolation function is unnecessary in a recently proposed covariant completion of Erik Verlinde's emergent gravity, and that Verlinde's approach moreover fixes the function's one free parameter. The so-derived correlation between the observed acceleration (inferred from rotation curves) and the gravitational acceleration due to merely the baryonic matter fits well with data. We then argue that the redshiftdependence of galactic rotation curves could offer a way to tell apart different versions of modified gravity from particle dark matter.
\end{abstract}

\section{Introduction}

It has been known for several decades that Modified Newtonian Dynamics (MOND) [1] explains some observed phenomena that have proved difficult to reproduce with particle dark matter. The most notable of these phenomena is the stunningly tight correlation between the gravitational pull which we observe acting on stars in galaxies - for example through rotation curves - and the gravitational pull that is caused by the 'normal' baryonic matter only [2]. While particle dark matter does a better job with the temperature fluctuations of the cosmic microwave background and its flexibility is of advantage to describe galaxy clusters, the successes of MOND on galactic scales are remarkable regardless [3].

MOND is a non-relativistic theory to which several relativistic completions are known [4, 5, 6, 7, 8, 9, 10, 11]. We here focus on a new member of this class, Covariant Emergent Gravity (CEG), which was recently proposed in [11]. We will show that CEG, unlike MOND, does not need an interpolation function but instead predicts a particular interpolation function. Moreover, if one follows the argument proposed in [12], this interpolation function has no free parameters. We demonstrate that the so-obtained equation fits the data well. 


\section{Modified Newtonian Gravity}

The defining equation of MOND is

$$
\vec{\nabla} \cdot\left(\mu\left(\left|\vec{\nabla} \phi_{\mathrm{MOND}} / a_{0}\right|\right) \vec{\nabla} \phi_{\mathrm{MOND}}\right)=4 \pi G \rho,
$$

where $G$ is Newton's constant, $\rho$ is the energy density of baryonic matter and $\phi_{\text {MOND }}$ is the modified Newtonian potential. The function $\mu$ is the interpolation function and $a_{0}$ quantifies an acceleration that is the theory's one free parameter. From comparison with data one finds that the following relation is approximately correct [13]:

$$
2 \pi a_{0} \approx H_{0} \approx \sqrt{\Lambda / 3}
$$

where $H_{0}$ is the Hubble constant and $\Lambda$ the cosmological constant. The numerical value is $a_{0} \approx 10^{-10} \mathrm{~m} / \mathrm{s}^{2}$.

The interpolation function is necessary to switch off the MOND effects and get back normal General Relativity in deep gravitational potentials. It is often taken to be of the form

$$
\mu(x)=\frac{x}{1+x},
$$

which does a good job to fit data on galactic sizes.

From $\mu$ one can calculate the relation between the total acceleration, $g_{\text {tot }}$, and the acceleration created by the baryonic ("normal") mass only, $g_{\mathrm{B}}$. This gives

$$
\mu\left(g_{\text {tot }} / a_{0}\right) \vec{g}_{\text {tot }}=\vec{g}_{\mathrm{B}},
$$

where $g_{\text {tot }}=\left|\vec{g}_{\text {tot }}\right|$. In the limit where $1 \ll g_{\text {tot }} / a_{0}$, ie accelerations are large compared to $a_{0}$, the interpolation function goes to 1 , so we recover the normal Newtonian limit. On the other hand, in the limit where $g_{\text {tot }} / a_{0} \ll 1$, ie accelerations are small compared to $a_{0}$, we are in the MOND regime where $g_{\text {tot }}^{2}=a_{0} g_{\mathrm{B}}$.

In the case of spherical symmetry, the solution to Eq. (1) in the MOND-regime is $\phi_{\text {MOND }} \sim \ln (r)$, as opposed to $\phi_{\text {MOND }} \sim 1 / r$ in the Newtonian limit. This means that in the MOND-regime the force acting on test-particles orbiting a large mass $M$ (eg the galactic center) is proportional to $1 / r$, resulting in flat rotation curves. It also follows that the total mass $M \sim v^{4}$, where $v$ is the limiting velocity of the rotation curves. This is the observationally well-established Tully-Fisher relation [14].

\section{Covariant Emergent Gravity}

The defining Lagrangian of CEG is that of matter coupled to gravity and an additional vector field $u_{\mu}$. In the non-relativistic limit it gives rise to the equation [11]

$$
\vec{\nabla} \cdot(|\vec{\nabla} \phi| \vec{\nabla} \phi)=\frac{2 \pi G}{3 L} \rho,
$$


where $L$ is a constant of dimension length (more about which later), and $\phi$ is proportional to the absolute value of the vector-field $u_{\mu}$.

At first sight, Eq. (5) looks pretty much the same as Eq. (1) except for the different constants. But they are not the same because the scalar $\phi$ in Eq. (5) is not the gravitational potential as in (1). Instead, this scalar causes an additional force acting on baryons by direct interaction. In CEG the normal gravitational potential $\phi_{\mathrm{N}}$ is instead determined, as usual, by

$$
\Delta \phi_{\mathrm{N}}=4 \pi G \rho .
$$

In CEG now the total acceleration, $g_{\text {tot }}$, which acts on baryons comes from the gradient of $\phi+\phi_{N}$, not from the gradient of $\phi$ alone, as in MOND. For a test-particle in a spherically symmetric field, (such as a star orbiting the galactic center), the total acceleration is

$$
g_{\text {tot }}=g_{\mathrm{B}}+g_{\phi} \quad \text { where } \quad g_{\mathrm{B}}=\partial_{r} \phi_{\mathrm{N}}, g_{\phi}=\partial_{r} \phi .
$$

In the case of spherical symmetry, it is straight-forward to use Eq. (7) to calculate the relation between the acceleration expected only from the baryonic mass, $g_{\mathrm{B}}$ and the total acceleration $g_{\text {tot }}$. In spherical coordinates, Eq. (5) reads

$$
\frac{1}{r^{2}} \partial_{r}\left(r^{2}\left(\partial_{r} \phi\right)^{2}\right)=\frac{2 \pi G}{3 L} \rho
$$

Multiplying this equation with $r^{2}$ and integrating it once yields

$$
r^{2}\left(\partial_{r} \phi\right)^{2}=\frac{G}{6 L} M(r) \quad \text { with } \quad M(r)=4 \pi \int_{0}^{r} d r^{\prime} r^{\prime 2} \rho\left(r^{\prime}\right) .
$$

If we now divide this equation by $r^{2}$, then the right side becomes just the normal gravitational acceleration of the baryonic mass, $g_{\mathrm{B}}$, while the left side is the square of the acceleration that comes from the interaction with the new field $g_{\phi}$. Taking the square root, we get

$$
g_{\phi}=\sqrt{\frac{g_{\mathrm{B}}}{6 L}} .
$$

This can now be inserted into (7) to give the 'radial acceleration relation':

$$
g_{\text {tot }}=g_{\mathrm{B}}\left(1+\sqrt{\frac{\tilde{a}_{0}}{g_{\mathrm{B}}}}\right),
$$

where $\tilde{a}_{0}:=1 /(6 L)$. Finally, by solving Eq. (11) for $g_{\mathrm{B}} / \tilde{a}_{0}$, we see that in the nonrelativistic limit CEG corresponds to MOND with the interpolation function

$$
\tilde{\mu}(x)=(1+2 x-\sqrt{1+4 x}) /(2 x) .
$$


The Redshift-Dependence of Radial Acceleration

Relation (11) was previously mentioned in [15]. The derivation we have presented here differs from the argument in [15] in two important points. First, our derivation is valid for general, spherically symmetric mass distributions and not merely for a point mass, as in [15]. Second, our result follows directly from a Lagrangian formulation and not from ad-hoc equations.

Let us then say something about the free constant $L$ which enters $\tilde{a}_{0}$. In [12], Verlinde fixes this constant by the following argument, hereafter referred to as 'Verlindematching.' The additional force acting on baryonic matter is caused by the change in entanglement entropy induced by the presence of the matter. This change comes about because inserting a baryonic mass into an asymptotic de-Sitter space slightly shifts the de-Sitter horizon, thereby changing the volume inside the horizon. Verlinde then requires that the horizon-shift induced by the presence of baryonic matter is identical to the shift quantified by the new field, which leads to $1 / L=\sqrt{\Lambda / 3}$ in a universe with $\Omega_{\Lambda}=1$ and $\Omega_{m}=0$, and $1 / L \approx 1.05 \times \sqrt{\Lambda / 3}$ in a universe with $\Omega_{\Lambda}=0.7$ and $\Omega_{m}=0.3$.

While this argument lacks rigor, the consequence is that in the non-relativistic limit, CEG with Verlinde-matching has no free parameters.

\section{Comparison with Observation}

Since a model without free parameters is every phenomenologist's nightmare, we now perform a sanity check and compare the radial acceleration relation (11) with observation. For this we use the data-set compiled in [2] which collects 2693 measurements from rotation curves of 153 galaxies.

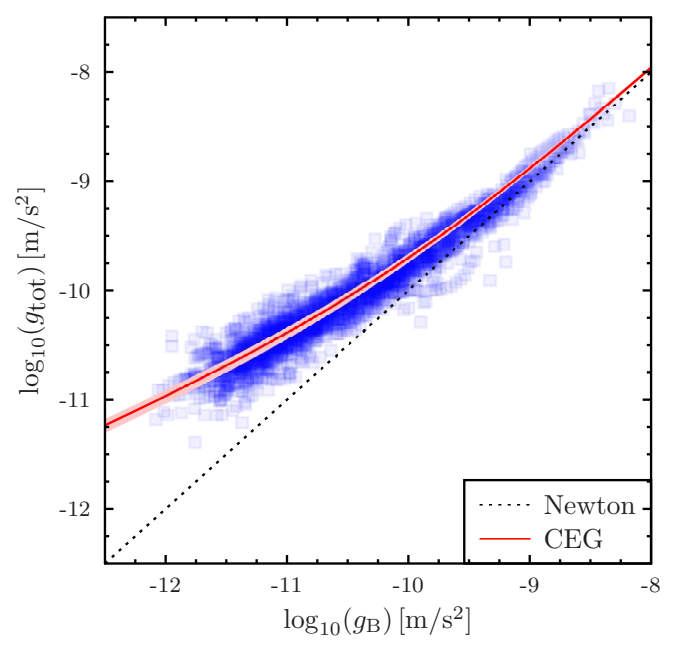

Figure 1: Observed, total acceleration $\left(g_{\text {tot }}\right)$ versus acceleration due to baryonic mass only $\left(g_{\mathrm{B}}\right)$. Blue squares are data from [2]. Red, solid curve: CEG with Verlinde-matching. Pink shading: $1 \sigma$ uncertainty. Dashed, black line: Newtonian gravity without dark matter.

For Figure 1 we have fixed $L$ using the Verlinde-matching as explained above. This gives the value $\tilde{a}_{0}=(0.96 \pm 0.01) 10^{-10} \mathrm{~m} / \mathrm{s}^{2}$ with the dominant error coming from the uncertainty in the Hubble rate [16]. We note in the passing that the equation derived here 
from CEG does not suffer from the problem with Verlinde's model pointed out in [17].

A $\chi^{2}$ fit gives the best-fit value $\tilde{a}_{0}=(0.77 \pm 0.01) \times 10^{-10} \mathrm{~m} / \mathrm{s}^{2}$, where the uncertainty is that of the statistical fit. We do not plot the curve with the best-fit value because by eye it cannot be distinguished from the curve with the Verlinde-matching. The statistical uncertainty on $\tilde{a}_{0}$ is small due to the large number of data points, but the data bring in a measurement error of $\sim 0.24 \times 10^{-10} \mathrm{~m} / \mathrm{s}^{2}$ from the normalization of the stellar massto-light ratio [2], which is the error depicted in Figure 1. We conclude that CEG with Verlinde-matching is consistent with data, at least so far.

We interpret CEG as a limit in which the additional field is in a superfluid phase. As laid out in [18], this limit no longer applies on the scale of solar systems (because the gradient of the field is too large) and also not on the scale of galaxy clusters (because the average potential isn't deep enough). Hence, one should not read too much into the fit at the lowest and highest accelerations.
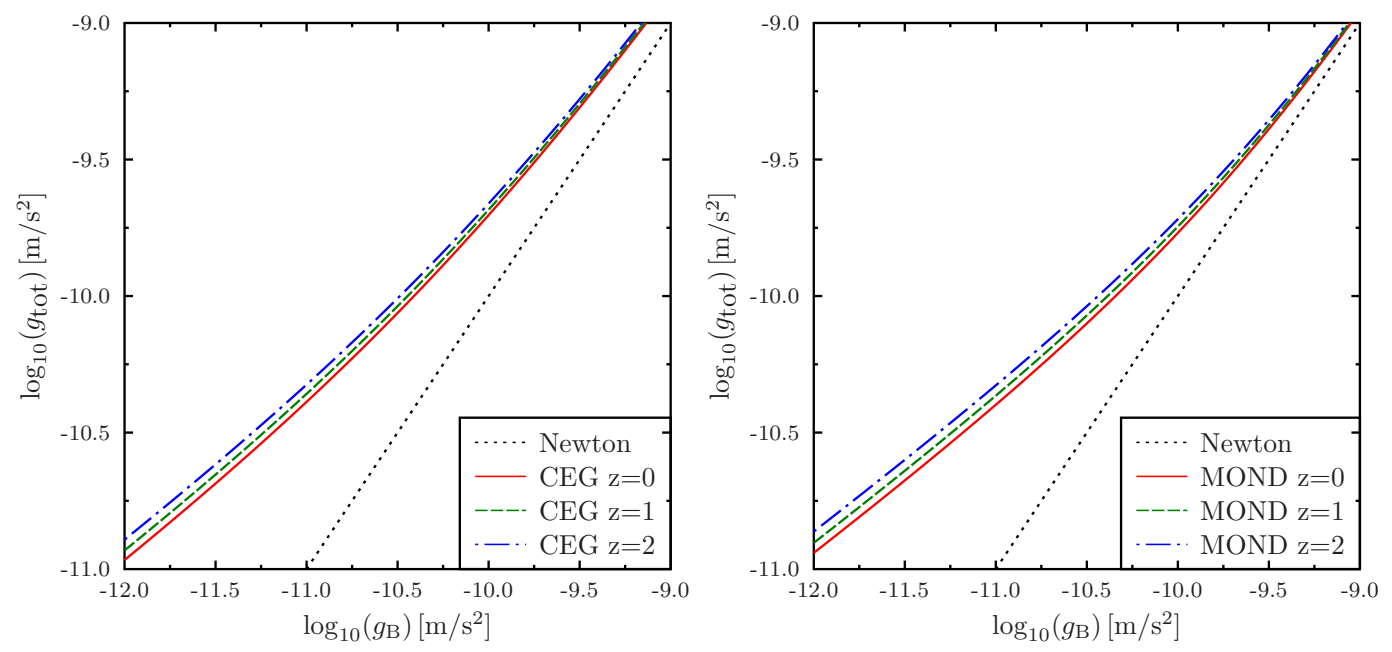

Figure 2: Redshift-dependence of radial acceleration relation for CEG and MOND.

Interestingly, however, as laid out earlier, Verlinde-matching relates $\tilde{a}_{0}$ with the size of the cosmological horizon which is a redshift-dependent quantity. We expect such a redshift-dependence in any approach that ties the acceleration scale to the de-Sitter temperature, as for example through the argument in [19]. It was even argued in [13] that MOND itself should have a redshift dependence.

This redshift-dependence induced through the changing size of the cosmological horizon is, however, small compared to that expected from particle dark matter. Figure 2 shows the redshift-dependence of CEG with Verlinde-matching and MOND. This can be compared to Figure 3 (from [20]) which was obtained from the McMaster Unbiased Galaxy Simulations and predicts a much larger redshift-dependence than that of modified gravity. 


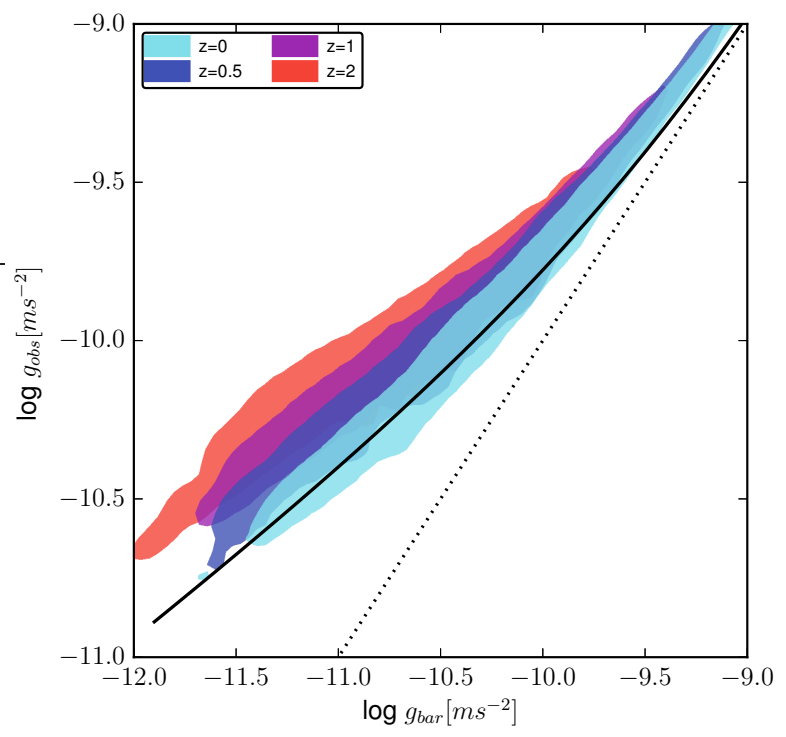

Figure 3: Redshift-dependence of the radial acceleration relation for particle dark matter based on the numerical simulation of [20].

Of course other numerical simulations might yield somewhat different results. Nevertheless we wish to propose here that, if data become better, the redshift-dependence of the radial acceleration could be used to tell apart modified gravity from particle dark matter.

\section{Acknowledgements}

We thank Ben Keller for the permission to reuse Figure 3 from [20].

\section{References}

[1] M. Milgrom, Astrophys. J. 270, 365 (1983).

[2] Stacy McGaugh, Federico Lelli, Jim Schombert, Phys. Rev. Lett. 117, 201101 (2016) arXiv:1609.05917 [astro-ph.GA].

[3] B. Famaey and S. McGaugh, Living Rev. Rel. 15, 10 (2012) arXiv:1112.3960 [astroph.CO]].

[4] J. Bekenstein and M. Milgrom, Astrophys. J. 286, 7 (1984).

[5] M. Milgrom, Mon. Not. Roy. Astron. Soc. 403, 886 (2010) doi:10.1111/j.13652966.2009.16184.x [arXiv:0911.5464[astro-ph.CO]].

[6] J. D. Bekenstein, Phys. Rev. D 70, 083509 (2004) Erratum: [Phys. Rev. D 71, 069901 (2005)] [astro-ph/0403694]. 
[7] T. G. Zlosnik, P. G. Ferreira and G. D. Starkman, Phys. Rev. D 75, 044017 (2007) [astro-ph/0607411].

[8] M. Milgrom, Phys. Rev. D 80, 123536 (2009) [arXiv:0912.0790 [gr-qc]].

[9] C. Deffayet, G. Esposito-Farese and R. P. Woodard, Phys. Rev. D 84, 124054 (2011) [arXiv:1106.4984 [gr-qc]].

[10] L. Blanchet and L. Heisenberg, JCAP 1512, no. 12, 026 (2015) arXiv:1505.05146 [hepth]].

[11] S. Hossenfelder, Phys. Rev. D 95, no. 12, 124018 (2017) [arXiv:1703.01415 [gr-qc]].

[12] E. P. Verlinde, SciPost Phys. 2, no. 3, 016 (2017) [arXiv:1611.02269 [hep-th]].

[13] M. Milgrom, arXiv:1703.06110 [astro-ph.GA].

[14] R. B. Tully and J. R. Fisher Astron. Astrophys. 54 (1977) 661-673.

[15] M. Milgrom and R. H. Sanders, arXiv:1612.09582 [astro-ph.GA].

[16] Planck Collaboration, A\&A 594, A13,arXiv:1502.01589 [astro-ph.CO]

[17] F. Lelli, S. S. McGaugh and J. M. Schombert, Mon. Not. Roy. Astron. Soc. 468, no. 1, L68 (2017) [arXiv:1702.04355 [astro-ph.GA]].

[18] L. Berezhiani and J. Khoury, Phys. Rev. D 92, 103510 (2015) [arXiv:1507.01019 [astroph.CO]].

[19] L. Smolin, Phys. Rev. D 96, no. 8, 083523 (2017) doi:10.1103/PhysRevD.96.083523 [arXiv:1704.00780 [gr-qc]].

[20] B. W. Keller, J. W. Wadsley, ApJL 835 L17 (2017) arXiv:1610.06183 [astro-ph.GA]. 